\title{
Application of central composite design for optimization of the removal of humic substances using coconut copra
}

\author{
Terri Zhuan Ean Lee ${ }^{1}$ Chanida Krongchai ${ }^{2} \cdot$ Nurul Aida Lu Mohd Irwn Lu ${ }^{1}$. \\ Sila Kittiwachana ${ }^{2} \cdot$ Siong Fong $\operatorname{Sim}^{1}$
}

Received: 10 February 2015/ Accepted: 2 June 2015/Published online: 19 June 2015

(c) The Author(s) 2015. This article is published with open access at Springerlink.com

\begin{abstract}
Coconut copra is a potential biosorbent for removal of humic substances from peat swamp runoff. In this paper, response surface methodology was applied to evaluate the optimum conditions for removal of humic substances from peat swamp runoff using modified coconut copra. Batch adsorption experiments were conducted according to central composite design. Results show that the quadratic model is best fitted for predicting the removal efficiency with regression coefficients closer to 1 and a lower root mean square error. Dosage is found to have significant influence on the removal efficiency with $p<0.05$. Response surface models further identified the optimum dosage, contact time and temperature at $4.56 \mathrm{~g}$ modified coconut copra per $100 \mathrm{~mL}$ peat swamp runoff, $42.9 \mathrm{~min}$ and $56.8^{\circ} \mathrm{C} / 329^{\circ} \mathrm{K}$, respectively attaining the maximum removal efficiency of $88.19 \%$. The predicted removal efficiency was confirmed experimentally under the modelled optimum conditions; the removal efficiency attained $(86.54 \%)$ was in good agreement with the predicted value.
\end{abstract}

Keywords Biosorbent · Coconut copra $\cdot$ Central composite design - Humic substances - Response surface methodology

Siong Fong Sim

sfsim@frst.unimas.my

1 Department of Chemistry, Faculty of Resource Science and Technology, Universiti Malaysia Sarawak, Kota Samarahan, Sarawak, Malaysia

2 Department of Chemistry, Faculty of Science, Chiang Mai University, Muang District, Chiang Mai, Thailand

\section{Introduction}

Humic substances are the products of biological and chemical decomposition of living organisms. They are found in abundance in black water and swampy areas, causing dark brown and yellowish colour in water. The presence of humic substances in water can have a significant impact on the treatability of the water and the efficiency of chemical disinfection process. They are believed to be the precursors to the formation of carcinogenic disinfection by-products. A previous study revealed that water tainted by peat swamp leachate contained a higher quantity of trihalomethanes compared to water from non-peat water sources [1].

In the conventional water treatment system, humic substances are removed using the coagulation and flocculation approach [2-4] requiring excess amount of coagulant and are generally limited by high operational and material costs. Other adsorbents such as activated carbon, zeolite, and mesoporous silica have also been employed for isolation of humic substances but these adsorbents are very expensive. Considering the cost and huge quantity of water treated, these adsorbents are less attractive. Biosorption is an alternative gaining increasing attention for its advantages of low cost and abundant availability. Sim et al. [5, 6] examined the adsorption ability of a spectrum of agricultural biomass in removing humic substances, and reported coconut copra with promising adsorption capacity. The biomass is relatively richer in carboxyl functional groups. The untreated coconut copra was evidenced to remove an average of $50 \%$ of humic substances from peat swamp runoff, higher than other selected biomasses including banana trunk, coconut husk, empty fruit bunch, groundnut shell, rice husk, sago waste, saw dust and sugarcane bagasse with removal efficiency ranging between 11 and $40 \%$, based on the spectrophotometric approach. Upon 
treatment with citric acid, the adsorption capability of coconut copra is greatly improved [6]. The heterogeneous nature of biomass and humic substances has allowed various interactions such as electrostatic linkages, cation bridging, hydrophobic interaction and hydrogen bonding to take place [7-9]. Coconut copra is also potentially viable for removal of heavy metals i.e., cadmium, chromium and nickel with encouraging removal efficiency of 85-90 \% [10]. This offers an environmental friendly and economically sustainable option for water and wastewater treatment.

It is very important to optimize the process conditions, minimizing the operational cost whilst maximizing the adsorption performance. According to Sim et al. [6], the adsorption performance of coconut copra for humic substances was optimized using the conventional one-factorat-a-time strategy; this involves changing a single factor whilst maintaining other factors at constant. This approach does not demonstrate the combined effect of all factors; in addition, it is tedious and provides insufficient information; often time, the optimization point could have been missed [11]. The traditional full factorial design can be used to examine the interaction effects nonetheless this usually involves a great number of experiments. For example, a typical three-factor-five-level full factorial design would require a total of 125 experimental runs while a corresponding central composite design (CCD) only involves 20 runs. CCD incorporates full or fractional factorial design with the centre and star points where each factor is mapped onto five-level allowing evaluation of the second-order response surface [12-15]. This design has been widely employed for process optimization involving dye removal [16-18], antibiotic production [19] and humic acid and heavy metal adsorption [20, 21]. Cerino Cordova et al. [22] conclude that it is necessary to study the interaction effects of various parameters on the optimal biosorption process. Therefore in this paper, inscribed CCD and response surface methodology were employed to examine the optimum conditions for removal of humic substances from peat swamp runoff using coconut copra. Note that this paper focuses on examining the optimum conditions for removal of humic substances when multiple variables are considered simultaneously; the information derived is particularly important for scaling up operation. The characteristics and adsorption isotherm studies of raw and modified coconut copra have been reported in Sim et al. [6].

\section{Materials and methods}

\section{Sample preparation}

Coconut copra in shredded form was collected from the local wet market and washed thoroughly under running tap water to remove coconut milk and foreign matters. The coconut copra was oven dried at $105{ }^{\circ} \mathrm{C}$. Coconut copra was then refluxed at $100{ }^{\circ} \mathrm{C}$ for one hour, washed, oven dried and subsequently agitated with $0.5 \mathrm{M}$ citric acid for one hour at room temperature. The treated coconut copra was washed to $\mathrm{pH} 3$ and oven dried [6].

\section{Adsorption of humic substances}

Peat swamp runoff, rich of humic substances, was collected from Asa Jaya Vicinity. Asa Jaya, located within Kota Samarahan Division, is surrounded with peat land and river is highly coloured. It was used to demonstrate the adsorption performance of treated coconut copra as the function of dosage, contact time and temperature in removing humic substances according to CCD. The adsorption was demonstrated on the 'real world' humic substances as the model of standard humic acid is incomparable to the naturally occurring humic as revealed [9]. Prior to adsorption test, peat swamp runoff at $\mathrm{pH} 4-5$ was filtered through $0.45 \mu \mathrm{m}$ membrane filter to remove suspended particles and foreign matter with no $\mathrm{pH}$ adjustment. The adsorption efficiency was examined based on the absorbance at $465 \mathrm{~nm}$ [23] using a UV-Visible spectrophotometer (Jasco V-360 Spectrophotometer). Hypothetically, the absorbance increases with the concentration of humic substances. When adsorption has taken place, reduction in absorbance is anticipated. The removal efficiency is calculated as follows.

\section{Removal Efficiency

$$
=\frac{\text { Abs of untreated water }- \text { Abs of treated water }}{\text { Abs of untreated water }} \times 100 \% \text {. }
$$

\section{Experimental design}

The CCD of three factors involving dosage $\left(x_{1}\right)$, contact time $\left(x_{2}\right)$ and temperature $\left(x_{3}\right)$ is shown in Fig. 1. The design consists of the factorial design with centre points and the star/axial points used to estimate the curvature. This allows the quadratic terms to interfere in the model for estimation of the optimum combination of factors. The total number of experiment is calculated based on $n=2 f+2 f+n$ where $f$ is the number of factors and $n$ is the replication of centre point [24]. The $\alpha$ value is defined as the distance between axial points and centre point, $\alpha=\left(2^{f}\right)^{\frac{1}{4}}$. As inscribed CCD is a scaled down of circumscribed CCD, the factorial design is further divided by $\alpha$, demonstrating a $1 / \alpha$ coded level in factorial design. Hence, coded value of 0.5946 is generated from $1 / 1.6818$. Table 1 summarizes the coded and uncoded level of the selected factors where the response is represented by the percentage 


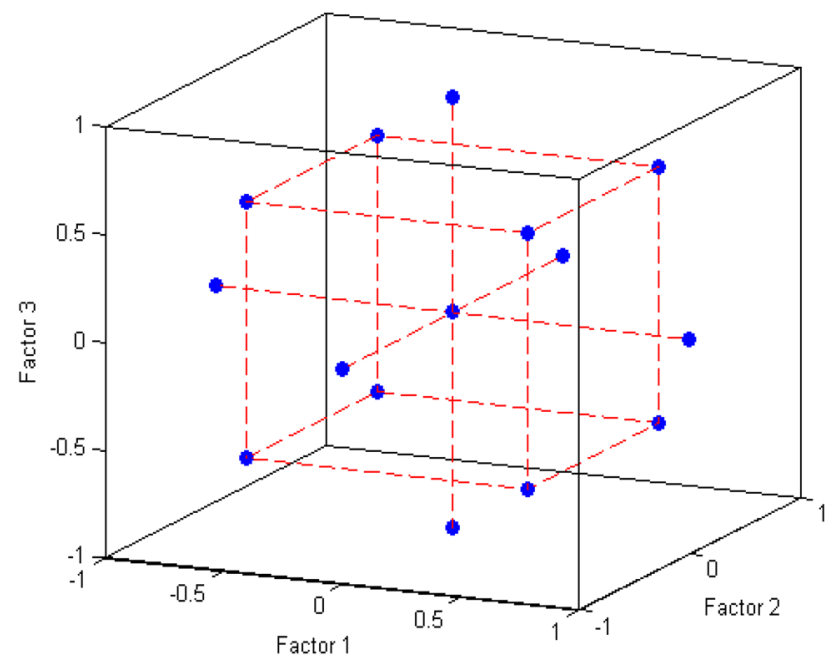

Fig. 1 The central composite design

removal of humic substances (Table 2). The CCD is more powerful for that the conventional fractional factorial designs can only be used to investigate the effect of a single variable on the response, unable to illustrate the optimum point.

\section{Response surface methodology (RSM)}

The design matrix involving linear, interaction and quadratic terms are employed to develop the equations for estimation of the adsorption performance. The variable coefficients were derived based on multiple linear regression: $\hat{\boldsymbol{y}}=\boldsymbol{D} \cdot \boldsymbol{b}$, where the predicted response is expressed as the product of the design matrix, $\boldsymbol{D}$, and the corresponding coefficients, $\boldsymbol{b}$ [25]. The vector of the corresponding coefficients is the pseudo inverse of $\boldsymbol{D}$ and the experimental response, $\boldsymbol{b}=\left(\boldsymbol{D}^{\prime} \cdot \boldsymbol{D}\right)^{-1} \cdot \boldsymbol{D}^{\prime} \cdot \boldsymbol{y}$. The coefficients indicate the behaviour of different factors on the response where various models can be described. The linear model:

$\hat{y}=b_{1} x_{1}+b_{2} x_{2}+b_{3} x_{3}+k$.

The interaction model:

$\hat{y}=b_{1} x_{1}+b_{2} x_{2}+b_{3} x_{3}+b_{12} x_{1} x_{2}+b_{13} x_{1} x_{3}+b_{23} x_{2} x_{3}+k$.
The quadratic model:

$\hat{y}=b_{1} x_{1}+b_{2} x_{2}+b_{3} x_{3}+b_{12} x_{1} x_{2}+b_{13} x_{1} x_{3}+b_{23} x_{2} x_{3}$

$+b_{1} x_{1}^{2}+b_{2} x_{2}^{2}+b_{3} x_{3}^{2}+k$,

where $\hat{y}=$ predicted response; $x_{1}, x_{2}$ and $x_{3}=$ coded factors; $b=$ coefficients; $k=$ offset term.

For a design with three or more levels, a curvature is observed. The response surface plots were generated using MATLAB version R2012a to enable modelling of the response over an entire range of varying factors with limited number experimental runs. Analysis of Variance (regression ANOVA) was employed to determine the model fitness and significance of various factors on the removal efficiency considering the sum of squares and residual sum of squares. ANOVA was in addition performed by differentiating the variances of linear regressions. All statistical analysis was performed using SPSS version 21.0.

\section{Model quality}

The regression coefficients, $R^{2}$ and adjusted $R^{2}$, were calculated to determine the variation between the model and the experimental data [22]. According to Weinberg and Abramowitz [26], $R^{2}$ often overestimates the model fit with excessive predictors therefore adjusted $R^{2}$ is recommended. The adjusted $R^{2}$ takes all independent predictors into consideration and fixes the $R^{2}$ more accurately according to the number of predictors present in the model. $R^{2}$ usually increases with increasing predictors; however, adjusted $R^{2}$ will only increase when that particular predictor has positive effects on the response. Thus, the latter is particularly useful in comparing models with different numbers of predictors [27]. Both $R^{2}$ and adjusted $R^{2}$ indicate the quality of the proposed models; values closer to 1.0 demonstrate a good agreement between the experimental and predicted responses [16]. The root mean square error (RMSE) and percentage root mean square error (\% RMSE) are also calculated to indicate the predictive performance [25, 28]. The lower is the value of RMSE, the better is the model for prediction.

$R^{2}=1-\frac{\sum_{i=1}^{I}(y-\hat{y})^{2}}{\sum_{i=1}^{I}(y-\bar{y})^{2}}$
Table 1 The coded and uncoded level of three selected factors

\begin{tabular}{lllllll}
\hline Factors & \multicolumn{7}{l}{ Coded levels } \\
\cline { 2 - 7 } & & -1 & $-\alpha(-0.59)$ & 0 & $\alpha(0.59)$ & 1 \\
\hline Dosage $(\mathrm{g})$ & $x_{1}$ & 1 & 1.82 & 3 & 4.18 & 5 \\
Contact time $(\min )$ & $x_{2}$ & 15 & 24.2 & 37.5 & 50.8 & 60 \\
Temperature $\left( \pm 5^{\circ} \mathrm{C} /{ }^{\circ} \mathrm{K}\right)$ & $x_{3}$ & $30 / 303$ & $40 / 313$ & $50 / 323$ & $60 / 333$ & $70 / 343$ \\
\hline
\end{tabular}


Table 2 The experimental and the predicted response according to linear, interaction and quadratic models

\begin{tabular}{|c|c|c|c|c|c|c|c|}
\hline \multirow[t]{2}{*}{ Experiment number $(n)$} & \multicolumn{3}{|c|}{ Experimental design } & \multicolumn{4}{|c|}{ Removal efficiency (\%) } \\
\hline & $x_{1}$ & $x_{2}$ & $x_{3}$ & Observed & Predicted (linear) & Predicted (interaction) & Predicted (quadratic) \\
\hline 1 & 1.82 & 24.2 & $40 / 313$ & 55.80 & 57.64 & 57.45 & 54.72 \\
\hline 2 & 1.82 & 24.2 & $60 / 333$ & 60.74 & 60.36 & 59.38 & 56.65 \\
\hline 3 & 1.82 & 50.8 & $40 / 313$ & 59.39 & 60.41 & 61.72 & 59.00 \\
\hline 4 & 1.82 & 50.8 & $60 / 333$ & 60.36 & 63.13 & 62.99 & 60.26 \\
\hline 5 & 4.18 & 24.2 & $40 / 313$ & 81.07 & 83.51 & 83.36 & 80.64 \\
\hline 6 & 4.18 & 24.2 & $60 / 333$ & 84.94 & 86.22 & 87.53 & 84.81 \\
\hline 7 & 4.18 & 50.8 & $40 / 313$ & 79.01 & 86.28 & 85.30 & 82.57 \\
\hline 8 & 4.18 & 50.8 & $60 / 333$ & 85.53 & 88.99 & 88.80 & 86.08 \\
\hline 9 & 1 & 37.5 & $50 / 323$ & 40.05 & 51.57 & 51.57 & 43.15 \\
\hline 10 & 5 & 37.5 & $50 / 323$ & 89.03 & 95.07 & 95.07 & 86.65 \\
\hline 11 & 3 & 15 & $50 / 323$ & 69.71 & 70.99 & 70.99 & 72.86 \\
\hline 12 & 3 & 60 & $50 / 323$ & 79.93 & 75.65 & 75.65 & 77.52 \\
\hline 13 & 3 & 37.5 & $30 / 303$ & 72.13 & 71.03 & 71.03 & 70.88 \\
\hline 14 & 3 & 37.5 & $70 / 343$ & 73.46 & 75.60 & 75.60 & 75.45 \\
\hline 15 & 3 & 37.5 & $50 / 343$ & 78.08 & 73.32 & 73.32 & 79.18 \\
\hline 16 & 3 & 37.5 & $50 / 343$ & 78.19 & 73.32 & 73.32 & 79.18 \\
\hline 17 & 3 & 37.5 & $50 / 343$ & 77.98 & 73.32 & 73.32 & 79.18 \\
\hline 18 & 3 & 37.5 & $50 / 343$ & 80.41 & 73.32 & 73.32 & 79.18 \\
\hline 19 & 3 & 37.5 & $50 / 343$ & 80.30 & 73.32 & 73.32 & 79.18 \\
\hline 20 & 3 & 37.5 & $50 / 343$ & 80.23 & 73.32 & 73.32 & 79.18 \\
\hline
\end{tabular}

$\operatorname{Adj} R^{2}=1-\frac{\left(1-R^{2}\right)(n-1)}{n-P-1}$

$\mathrm{RMSE}=\sqrt{\frac{\sum_{i=1}^{I}(y-\hat{y})^{2}}{n-P}}$

$\% \mathrm{RMSE}=\sqrt{\frac{\sum_{i=1}^{I}(y-\hat{y})^{2}}{n-P}} \times \frac{100 \times n}{\sum_{i=1}^{I} y}$,

where $n=$ number of experiments; $P=$ number of predictors.

\section{Results and discussion}

Dosage, contact time and temperature are three major factors governing the adsorption performance of biosorbents [17, 18]. With CCD, these factors can be systematically optimized taken into account of their interaction effects. The linear, interaction and quadratic models were derived based on CCD where the magnitudes of coefficients suggest the contribution of various factors on the response. It is evidenced that dosage exerts greater influence on the removal efficiency in all models.

Linear model:

$\hat{y}=21.752 x_{1}+2.331 x_{2}+2.284 x_{3}+71.357$.

Interaction model:

$\hat{y}=21.752 x_{1}+2.331 x_{2}+2.284 x_{3}-1.654 x_{1} x_{2}$

$$
+1.586 x_{1} x_{3}-0.470 x_{2} x_{3}+71.357 \text {. }
$$

Quadratic model:

$$
\begin{aligned}
\hat{y}= & 21.752 x_{1}+2.331 x_{2}+2.284 x_{3}-1.654 x_{1} x_{2} \\
& +1.586 x_{1} x_{3}-0.470 x_{2} x_{3}-14.166 x_{1}^{2}-3.889 x_{2}^{2} \\
& -5.912 x_{3}^{2}+79.072 .
\end{aligned}
$$

Table 2 summarizes the predicted response according to different models. The $R^{2}$ value calculated for linear, interaction and quadratic models are 0.825, 0.827 and 0.973 , respectively. Meanwhile, the corresponding adjusted $R^{2}$ values are $0.793,0.748$ and 0.949 implying that the adsorption process is more suitably described with the quadratic model. 
Table 3 ANOVA regression models for removal of humic substances

\begin{tabular}{lccccc}
\hline & Sum of squares & Degree of freedom & Mean square & F & $p$ \\
\hline Linear model & & & & & \\
Regression & 2336.032 & 3 & 778.677 & 25.205 & 0.000 \\
Residual & 494.307 & 16 & 30.894 & & \\
Interaction model & & & & & \\
Regression & 2341.503 & 6 & 390.251 & 10.378 & 0.000 \\
Residual & 488.835 & 13 & 37.603 & & \\
Quadratic model & & & 306.123 & 40.692 & 0.000 \\
Regression & 2755.109 & 9 & 7.523 & & \\
Residual & 75.229 & 10 & & & \\
Total & 2830.338 & 19 & & &
\end{tabular}

The deviation between observed and predicted percentage according to linear, interaction and quadratic models were $0.63-28.76,1.53-28.76$ and $0.15-7.74 \%$ suggesting that the quadratic model has the lowest error. RMSE serves as a measure of predictive power based on difference between observed and predicted values. The RMSE calculated according to linear, interaction and quadratic models are $5.39,5.91$ and 2.62, respectively. These values can be converted into \% RMSE in turn to indicate the closeness of the predicted and experimental responses. The corresponding $\%$ RMSE are $7.35 \%$ (linear), $8.06 \%$ (interaction) and $3.57 \%$ (quadratic). This suggests that the quadratic model is best fitted for estimation of the removal efficiency. Noticeably, the interactions terms are relatively smaller, indicative of less influence on the adsorption.

The fitness of the model was examined using regression ANOVA [17]. Under the null hypothesis, the model has no predictive capability. The $p$ values obtained for linear, interaction and quadratic models are consistently $<0.05$ (Table 3) suggesting that these models are capable of predicting the response. Table 4 shows the estimated regression coefficients and the corresponding $t$ and $p$ values; all linear, interaction and quadratic terms are characterized by $p>0.05$ except $x_{1}$ and $x_{1}^{2}$ indicating that dosage has a significant effect. Factor $x_{3}$ demonstrates non-significant linear term but significant quadratic term. This is possibly due to the response relies more on the quadratic form rather than linear form or in other words, $x_{3}$ has a quadratic effects towards the response. Figure 2 illustrates the interaction plot of dosage, contact time and temperature on the removal efficiency of humic substances. Typically, if there is no interaction, the profile of respective factor would appear parallel.

Figure 3 illustrates the three-dimensional response surface plots of removal efficiency against two factors. The surface plots of (dosage vs temperature) and (dosage vs contact time) indicate that at higher dosage, the adsorption process is favoured attaining elevated removal efficiency possibly due to the increased binding sites. The optimum dosage is found at $4.56 \mathrm{~g} / 100 \mathrm{~mL}$. The removal efficiency
Table 4 Estimated regression coefficients and the corresponding $t$ and $p$ values

\begin{tabular}{lrlrl}
\hline Term & Coefficient & Standard deviation & \multicolumn{1}{l}{$t$} & $p$ \\
\hline Constant & 79.176 & 1.119 & 70.779 & 0.000 \\
$x_{1}$ & 21.752 & 1.248 & 17.427 & 0.000 \\
$x_{2}$ & 2.331 & 1.248 & 1.867 & 0.091 \\
$x_{3}$ & 2.284 & 1.248 & 1.830 & 0.097 \\
$x_{1} x_{2}$ & -1.654 & 2.743 & -0.603 & 0.560 \\
$x_{1} x_{3}$ & 1.586 & 2.743 & 0.578 & 0.576 \\
$x_{2} x_{3}$ & -0.470 & 2.743 & -0.171 & 0.867 \\
$x_{1}^{2}$ & -14.267 & 2.044 & -6.981 & 0.000 \\
$x_{2}^{2}$ & -3.989 & 2.044 & -1.952 & 0.079 \\
$x_{3}^{2}$ & -6.012 & 2.044 & -2.942 & 0.015 \\
\hline
\end{tabular}

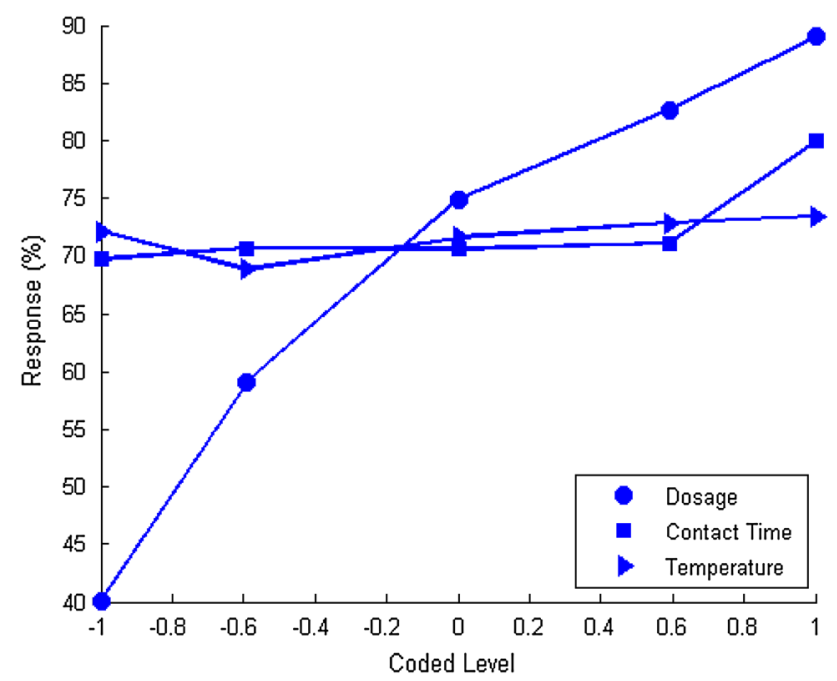

Fig. 2 The interaction plot of dosage, contact time and temperature

is in addition improved with increased temperature and contact time, though not as significant as the dosage, corroborating the coefficient values derived for respective 


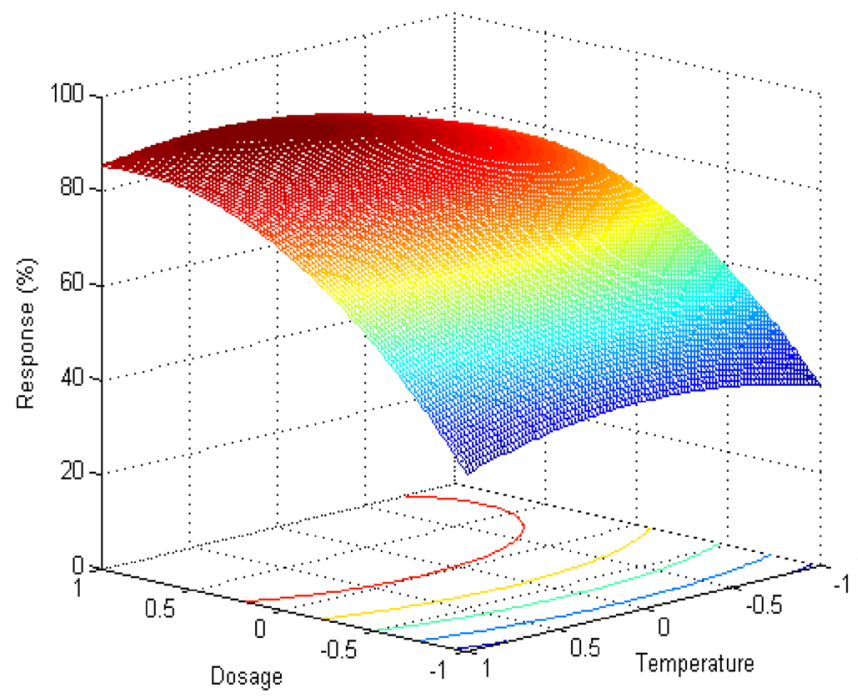

(a)

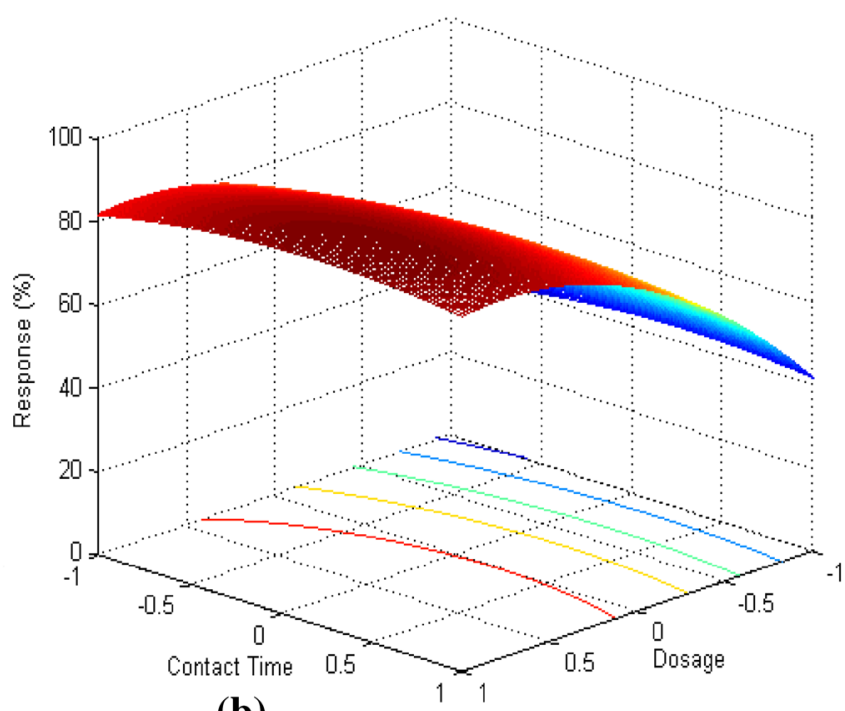

(b)

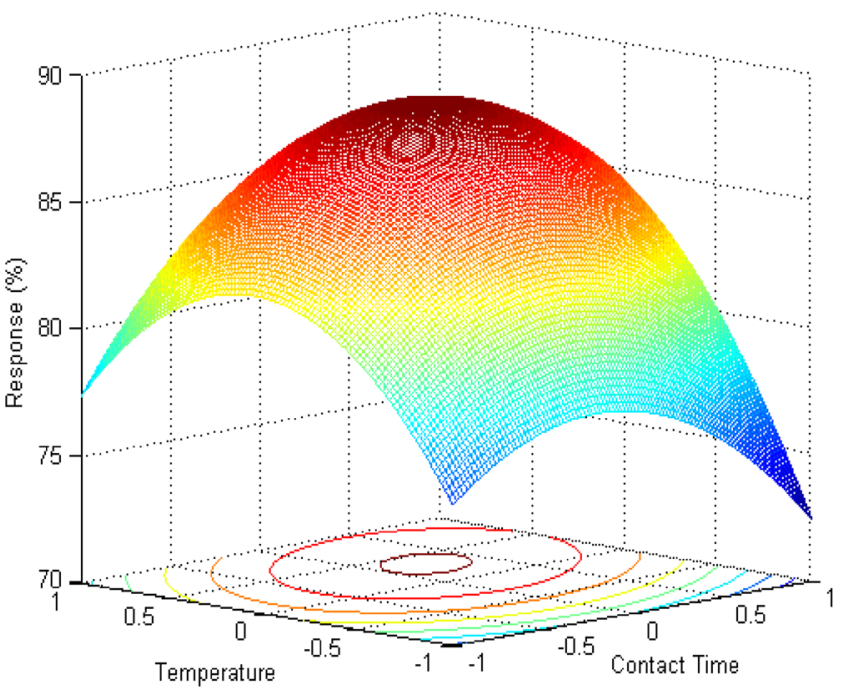

(c)

Fig. 3 The three-dimensional response surface plots of a response versus dosage and temperature; $\mathbf{b}$ response vs contact time and dosage; and c response vs temperature and contact time

factors based on the quadratic model $\left(b_{\text {dosage }}: 21.75 ; b_{\text {con- }}\right.$ tact time: $2.33 ; b_{\text {temperature }}: 2.28$ ). According to Yasin et al. [21], higher temperature may facilitate adsorption by speeding up the collision of particles and lowering the mass transfer energy. The optimal temperature identified for maximum removal percentage is $56.8^{\circ} \mathrm{C} / 329^{\circ} \mathrm{K}$, beyond which the removal efficiency begins to decline. This is possibly associated to the exothermic characteristic where desorption process is anticipated to take place $[17,29]$. The adsorption process is enhanced at longer contact time nevertheless the process appears to slow down after $43 \mathrm{~min}$ when the equilibrium is reached [17].

The maximum removal efficiency of $88.19 \%$ is attained when $4.56 \mathrm{~g}$ of coconut copra is agitated in $100 \mathrm{~mL}$ of peat swamp runoff for $42.9 \mathrm{~min}$ at $56.8{ }^{\circ} \mathrm{C} / 329^{\circ} \mathrm{K}$. The optimum conditions are experimentally verified where an average of $86.54 \%$ of removal is achieved corresponding to percentage error of $1.87 \%$. Yasin et al. [21] reported $73.46 \%$ removal with humic acid standard solution using anionic clay hydrotalcite under optimum conditions.

\section{Conclusion}

Coconut copra is a potential biosorbent for water and wastewater treatment. The quadratic model involving dosage, contact time and temperature is best fitted to predict the efficiency of coconut copra in removing humic 
substances from peat swamp runoff. Among these factors, dosage exhibits relatively greater influence. The optimal conditions necessary to attain maximum removal efficiency $(88.19 \%)$ are dosage (4.56 g modified coconut copra per $100 \mathrm{~mL}$ peat swamp runoff), temperature $\left(56.8^{\circ} \mathrm{C} / 329^{\circ} \mathrm{K}\right)$ and contact time $(42.9 \mathrm{~min})$. The experimental response acquired $(86.54 \%)$ based on the modelled optimum conditions is in good agreement with the predicted response. Coconut copra adsorbed with humic compounds can be potentially recycled into soil conditioner due to its beneficial agricultural value. Lee et al. [30] revealed that biomass fortified with humic compounds is a viable soil conditioner for improving plant growth.

Acknowledgments The authors would like to thank the Ministry of Science and Technology, Malaysia for funding this project (06-01-09SF0086). The authors also thank the industrial attachment scholarship provided by the Development and Promotion of Science and Technology Talents Project (DPST), Thailand.

Conflict of interest The authors declare that there are no competing interests.

Open Access This article is distributed under the terms of the Creative Commons Attribution 4.0 International License (http://creativecommons.org/licenses/by/4.0/), which permits unrestricted use, distribution, and reproduction in any medium, provided you give appropriate credit to the original author(s) and the source, provide a link to the Creative Commons license, and indicate if changes were made.

\section{References}

1. Sim SF, Mohamed M (2005) Occurrence of trihalomethanes in drinking water tainted by peat swamp runoff in Sarawak. J Sci Technol Trop 1:135-140

2. Amokrane A, Comel C, Veron J (1997) Landfill leachate pretreatment by coagulation-flocculation. Water Res 31:2775-2782

3. Liu X, Li XM, Yang Q et al (2012) Landfill leachate pretreatment by coagulation-flocculation process using iron-based coagulants: optimization by response surface methodology. Chem Eng J 200-202:39-51

4. Libecki B, Dziejowski J (2008) Optimization of humic acids coagulation with aluminum and Iron(III) salts. Polish J Environ Stud 17:397-403

5. Sim SF, Mohd Irwan LuNAL, Lee TZE, Mohamed M (2015) Removal of dissolved organic carbon from peat swamp runoff using assorted tropical agriculture biomass. Pertanika J Sci Technol 23:51-59

6. Sim SF, Lee TZE, Mohd Irwan LuNAL, Mohamed M (2014) Modified coconut copra residues as a low cost biosorbent for adsorption of humic substances from peat swamp runoff. Bioresources 9:952-968

7. Bai R, Zhang X (2001) Polypyrrole-coated granules for humic acid removal. J Colloid Interface Sci 243:52-60

8. Daifullah AAM, Girgis BS, Gad HMH (2004) A study of the factors affecting the removal of humic acid by activated carbon prepared from biomass material. Colloids Surf A 235:1-10

9. Deng S, Bai RB (2003) Aminated polyacrylonitrile fibers for humic acid adsorption: behaviors and mechanisms. Environ Sci Technol 37:5799-5805
10. Lee TZE, Mohd Irwn Lu NAL, Sim SF, Bakeh T (2013) Application of modified coconut copra as biosorbent for wastewater treatment. In: 26th Symposium of Malaysia Analytical Science Proceedings

11. Stoyanov K, Walmsley AD (2006) Response-surface modeling and experimental design. In: Gemperline P (ed) Practical Guidlines to Chemotherapy, 2nd edn. CRC Press, Florida, pp 290-294

12. Hibbert DB (2007) Quality assurance in the analytical chemistry laboratory. Oxford University Press, Oxford

13. Lee CP, Lo Huang MN (2011) D-optimal designs for secondorder response surface models with qualitative factors. J Data Sci 9:139-153

14. Otto M (2007) Chemometrics: statistics and computer application in analytical chemistry, 2nd edn. Wiley-VCH, Weinheim

15. Park SH, Kim HJ, Cho JI (2008) Optimal central composite designs for fitting second order response regression models. In: Shalabh, Heumann C (eds) Recent advances in linear models and related areas. pp 323-339

16. Demirel M, Kayan B (2012) Application of response surface methodology and central composite design for the optimization of textile dye degradation by wet air oxidation. Int J Ind Chem 3:1-10

17. Narayana Saibaba KV, King P, Gopinadh R, Sreelakshmi V (2011) Response surface optimization of dye removal by using waste prawn shells. Int J Chem Sci Appl 2:186-193

18. Ponnusamy SK, Subramaniam R (2013) Process optimization studies of Congo red dye adsorption onto cashew nut shell using response surface methodology. Int J Ind Chem 4:1-10

19. Smitha SL, Philip R (2014) Antibiotic production by a marine fungus Penicillium citrinum S36 through solid state fermentation : optimization by response surface methodology. Int $\mathrm{J}$ Res Biomed Biotechnol 4:6-13

20. Yasin Y, Mohamad M, Ahmad FB (2013) The application of response surface methodology for lead ion removal from aqueous solution using intercalated tartrate-Mg-Al layered double hydroxides. Int J Chem Eng 2013:1-7. doi10.1155/2013/937675

21. Yasin Y, Abdul Malek AH, Ahmad FB (2010) Response surface methodology study on removal of humic acid from aqueous solutions using anionic clay hydrotalcite. J Appl Sci 10:2297-2303

22. Cerino Cordova FJ, Garcia Leon AM, Garcia Reyes RB et al (2011) Response surface methodology for lead biosorption on Aspergillus terreus. Int J Environ Sci Technol 8:695-704

23. Hautala K, Peuravuori J, Pihlaja K (2000) Measurement of aquatic humus content by spectroscopic analyses. Water Res 34:246-258

24. Morgan ED (1991) Chemometrics: experimental design. Wiley, New York

25. Brereton RG (2003) Chemometrics: data analysis for the laboratory and chemical plant. Wiley, England

26. Weinberg SL, Abramowitz SK (2008) Statistics using SPSS: an integrative approach, 2nd edn. Cambridge University Press, Cambridge

27. Frost J (2013) Multiple regression analysis: Use adjusted $\mathrm{R}$-squared and predicted R-squared to include the correct number of variables. In: Minitab Blog

28. Lei $\mathrm{P}$ (1998) a linear programming method for synthesizing origin-destination (O-D) trip tables from traffic counts for inconsistent systems. Virginia Polytechnic Institute and State University, Blacksburg

29. Ravikumar K, Deebika B, Balu K (2005) Decolourization of aqueous dye solutions by a novel adsorbent: application of statistical designs and surface plots for the optimization and regression analysis. J Hazard Mater 122:75-83

30. Lee TZE, Mohd Irwan LuNAL, Mohd Razak NN et al (2014) Application of rice husk as water treatment agent and soil conditioner. Br J Appl Sci Technol 4:4252-4262 